\title{
Filmography
}

Most of the information included in this filmography comes either from the films themselves or from publicity material issued by production companies. Supplementary sources of information include journals Film Dope and The Monthly Film Bulletin as well as Denis Gifford's invaluable The British Film Catalogue 18951970: A Guide to Entertainment Films (Newton Abbot, David \& Charles, 1973) and Dave Rogers' The ITV Encyclopedia of Adventure (London, Boxtree, 1988).

\section{Editorial credits}

Terence Fisher worked as an editor from 1936 onwards, first at Gainsborough and subsequently at Warner Brothers-First National with some work done elsewhere for less well-known companies such as Greenspan \& Seligman and British Aviation. What follows is an indicative rather than a definitive listing of his credits. Given that so many films and associated records from this period are apparently lost, it seems unlikely that an authoritatively complete list of Fisher's work as an editor will ever be possible.

For Gainsborough: Jack of All Trades (1936, d. Jack Hulbert/Robert Stevenson), Tudor Rose (1936, d. Robert Stevenson), Where There's a Will (1936, d. William Beaudine), Everybody Dance (1936, d. Charles Reisner), Windbag the Sailor (1936, d. William Beaudine) - and, after some years away from the studio, The Wicked Lady (1945, d. Leslie Arliss).

For Warner Brothers-First National: Mr Satan (1938, d. Arthur Woods), George and Margaret (1940, d. George King), Atlantic 
Ferry (1941, d. Walter Forde), Flying Fortress (1942, d. Walter Forde), The Peterville Diamond (1942, d. Walter Forde), The Night Invader (1942, d. Herbert Mason), The Dark Tower (1943, d. John Harlow), The Hundred Pound Window (1943, d. Brian Desmond Hurst), Flight from Folly (1944, d. Herbert Mason).

Other credits included On the Night of the Fire (1939, d. Brian Desmond Hurst) for Greenspan \& Seligman, The 7 th Survivor (1941, d. Leslie Hiscott) for British National-Shaftesbury, Tomorrow We Live (1942, d. George King) and Candlelight in Algeria (1943, d. George King) for British Aviation, One Exciting Night (1944, d. Walter Forde) for Columbia British, and, as Fisher's final editorial credit, The Master of Bankdam (1947, d. Walter Forde) for Holbein.

\section{Television credits}

As with his editing credits, a definitive listing of Fisher's television work is probably not possible, and while researching this book I was unable to track down any of his TV work for viewing. The series upon which he worked included Douglas Fairbanks Presents (1953-1957), Colonel March Of Scotland Yard (1956-195 7), The Adventures of Robin Hood (1956-1960) - the episodes (all from Seasons 1 and 2) 'Trial by Battle', 'The Traitor', 'The Thorkill Ghost', 'Ransom', 'The Hero', 'Hubert', 'The Dream', 'The Blackbird', 'The Path of True Love' and 'The Infidel' - Assignment Foreign Legion (1957), The Gay Cavalier (1957) - the episodes 'Springtime for Julia', 'The Sealed Knot' and 'Girl of Quality' - Dial 999 (1958-1959) and Sword of Freedom (19591961). Of these, Colonel March Of Scotland Yard sounds the most intriguing. Based on stories by John Dickson Carr and starring Boris Karloff as the eponymous Colonel, its narratives seem to have contained pronounced supernatural elements.

\section{Directorial credits}

Film dates refer to the film's initial British release rather than the date of its production.

I have decided not to include in the filmography The Gelignite Gang (aka The Dynamiters, 1956) although it is sometimes attributed to Fisher, notably by Denis Gifford in The 
British Film Catalogue 1895-1970. Other sources attribute it as a co-directed project to Fisher and Francis Searle. In The Charm of Evil: The Life and Films of Terence Fisher (Metuchen, NJ and London, Scarecrow Press), 1991, p. 218, Wheeler Winston Dixon speculates that Fisher might have done some work on the film, although he does this on the basis of seeing a version of the film lacking a directorial credit. The version I have seen does have a directorial credit; it reads 'Directed by Francis Searle'. In a recent interview - in Brian McFarlane's An Autobiography of British Cinema (London, Methuen), 1997, pp. 522-6 - Searle himself has reminisced briefly about the film's production and given no indication that the film was co-directed. In the absence of hard evidence to the contrary, one has to suppose that in this case the usually ultrareliable Gifford made a mistake (his entry on the film does not even mention Searle).

However, I have included Three's Company - which contains material shot by Fisher for the television series Douglas Fairbanks Presents - because it did achieve a British theatrical release.

Colonel Bogey 1947, 51 min.

Production company/studio: Highbury

Producer: John Croydon

Screenplay: John Baines, W. E. C. Fairchild

Photography: Gordon Lang

Editor: Gordon Pilkington

Art director: Don Russell

Music: Norman Fulton

Cast: Mary Jerrold (Aunt Mabel), Jane Barrett (Alice Graham), Jack Train (Uncle James), John Stone (Wilfred Barriteau), Hedli Anderson (Millicent), Ethel Coleridge (Emily)

To The Public Danger 1948, $44 \mathrm{~min}$.

Production company/studio: Highbury

Producer: John Croydon

Screenplay: T. J. Morrison and Arthur Reid from Patrick

Hamilton's radio play

Photography: Harry Waxman, Roy Fogwell

Editor: Graeme Hamilton

Production designer: Ken Sharp 
Music: Doreen Carwithen

Cast: Dermot Walsh (Captain Cole), Susan Shaw (Nancy Bedford), Roy Plomley (Reggie), Barry Letts (Fred Lane), Betty Ann Davies (Barmaid), Patricia Hayes (Postmistress), Sam Kydd (Police Driver), John Lorrell (Police Sergeant), Frederick Piper (Labourer), Patience Rentoul (Labourer's wife)

\section{Song For Tomorrow 1948, 62 min.}

Production company/studio: Highbury

Producer: Ralph Nunn-May

Screenplay: W. E. C. Fairchild.

Photography: Walter J. Harvey

Editor: Gordon Pilkington

Art director: Donald Russell

Music: William Blezard

Cast: Evelyn McCabe (Helen Maxwell), Ralph Michael (Roger Stanton), Shaun Noble (Derek Wardell), James Hayter (Nicholas Klausmann), Valentine Dunn (Mrs Wardell), Christopher Lee (Auguste), Ethel Coleridge (Woman in cinema), Yvonne Foster (Nurse), Carleen Lord (Helen's dresser), Conrad Phillips (Lieutenant Fenton), Martin Boddey (Major), Sam Kydd (Sergeant), Lockwood West (Mr Stokes)

\section{Portrait From Life 1948, 90 min.}

Production company: Gainsborough

Studio: Shepherd's Bush

Producer: Antony Darnborough

Screenplay: Frank Harvey Jnr., Muriel Box and Sydney Box, from an original story by David Evans

Photography: Jack Asher

Editor: V. Sagovsky

Supervising art director: George Provis

Music: Benjamin Frankel

Cast: Guy Rolfe (Lawrence), Mai Zetterling (Hildegard/Lydia), Robert Beatty (Campbell Reid), Herbert Lom (Hendlmann), Arnold Marie (Menzel) and in order of appearance Peter Murray (Lieut. Keith), Thora Hird (Mrs Skinner), Eric Messiter (Coroner), Cyril Chamberlain (Supervisor), Patrick Holt (Ferguson), Betty Lynne (Woman Interpreter), Dorothea Glade 
(Hildegarde Schmidt), Nellie Arno (Anna Skutetsky), Richard Molinas (Man in crowd), Hugo Schuster (Interpreter), Gordon Bell (Capt. Roberts), Sam Kydd (Army Truck Driver), Gerard Heinz (Heine), Sybilla Binder (Eitel), George Thorpe (Brigadier), Philo Hauser (Hans), Eric Pohlmann (Leader of search party)

Marry Me! 1949, 97 min.

Production company: Gainsborough

Producer: Betty Box

Screenplay: Denis Waldock, Lewis Gilbert

Photography: Ray Elton

Editor: Gordon Pilkington

Art director: George Provis

Music: Clifton Parker

Cast: Derek Bond (Andrew Scott), Susan Shaw (Pat Cooper), Patrick Holt (Martin Roberts), Carol Marsh (Doris Pearson), David Tomlinson (David Haig), Zena Marshall (Marcelle), Guy Middleton (Sir George Blake), Nora Swinburne (Enid Lawson), Brenda Bruce (Brenda Delamere), Jean Cadell (Hester Parsons), Mary Jerrold (Emily Parsons), Denis O'Dea (Saunders), Yvonne Owen (Sue Carson), Alison Leggatt (Miss Beamish), Beatrice Varley (Mrs Perrins), Cyril Chamberlain (PC Jackson), Hal Osmond (Man in restaurant), Russell Waters (Mr Pearson), Joan Hickson (Mrs Pearson), Marianne Stone (Elsie), J. H. Roberts (Old Gent in Train), Lyn Evans (Railway Official), Anthony Steel (Jack Harris)

The Astonished Heart 1949, 89 min.

(co-directed with Antony Darnborough)

Production company: Gainsborough

Studio: Pinewood

Producer: Antony Darnborough

Screenplay: Noel Coward from his own play

Photography: Jack Asher

Editor: V. Sagovsky

Supervising art director: George Provis

Music: Noel Coward 
Cast: Noel Coward (Christian Faber), Celia Johnson (Barbara Faber), Margaret Leighton (Leonora Vail), Joyce Carey (Susan Birch), Graham Payn (Tim Verney), Amy Veness (Alice Smith), Ralph Michael (Philip Lucas), Michael Hordern (Ernest), Patricia Glyn (Helen), Alan Webb (Sir Reginald), Everly Gregg (Miss Harper), John Salew (Soames), Gerald Anderson (Waiter), John Warren (Barman)

So Long at the Fair 1950, $86 \mathrm{~min}$.

(co-directed with Antony Darnborough)

Production company: Gainsborough

Studio: Pinewood

Producer: Betty E. Box

Screenplay: Hugh Mills, Anthony Thorne

Photography: Reginald Wyer

Editor: Gordon Hales

Supervising art director: George Provis

Music: Benjamin Frankel

Cast: Jean Simmons (Vicky Barton), Dirk Bogarde (George Hathaway), David Tomlinson (Johnny Barton), Honor Blackman (Rhoda O'Donovan), Cathleen Nesbitt (Madame Herve), Felix Aylmer (Consul), Betty Warren (Mrs O'Donovan), Marcel Poncin (Narcisse), Austin Trevor (Commissaire), Andre Morell (Dr Hart), Zena Marshall (Nina), Eugene Deckers (Day Porter)

Home to Danger 1951, $66 \mathrm{~min}$.

Production company: New World/Lance Comfort Productions

Producer: Lance Comfort

Screenplay: Francis Edge, John Temple-Smith

Photography: Reg Wyer

Editor: Francis Edge

Art director: Cedric Dawe

Music: M. Arnold

Cast: Rona Anderson (Barbara), Guy Rolfe (Robert), Francis Lister

(Wainwright), Alan Wheatley (Hughes), Stanley Baker (Willie Dougan), Denis Harkin (Jimmy-the-one), Bruce Belfrage (Solicitor) 
The Last Page 1952, 84 min.

Production company: Exclusive-Lippert (Hammer)

Studio: Bray

Producer: Anthony Hinds

Screenplay: Frederick Knott from a play by James Hadley Chase Photography: Walter Harvey

Editor: Maurice Rootes

Cast: George Brent (Harman), Marguerite Chapman (Stella), Raymond Huntley (Clive), Peter Reynolds (Jeff), Diana Dors (Ruby), Eleanor Summerfield (Vi), Meredith Edwards (Dale), Isobel Dean (May Harman), Harry Fowler (Joe), Nelly Arno (Miss Rosetti), Conrad Phillips (Todd), Lawrence Ward (Lang), David Keir (Mr Quince), Eleanore Bryan (Mary), Jack Faint (Receptionist), Harold Goodwin (Waiter), Leslie Weston ( $\mathrm{Mr}$ Bruce), Courtney Hope (Woman Customer), John Mann (The Tobacconist), Archie Duncan (PC Gibbons), Lawrence O'Madden (Elderly Customer), Ian Wilson (Second Customer)

Wings of Danger 1952, $73 \mathrm{~min}$.

Production company: Hammer

Studio: Riverside

Producer: Anthony Hinds

Screenplay: John Gilling from the novel Dead on Course by Elleston Trevor and Packham Webb

Photography: Walter Harvey

Editor: Jim Needs

Art director: Andrew Mazzei

Musical director: Malcolm Arnold

Cast: Zachary Scott (Vaness), Robert Beatty (Nick), Naomi Chance (Avril), Arthur Lane (Boyd), Kay Kendall (Alexia), Colin Tapley (Maxwell), Harold Lang (Snell), Diana Cilento (Jeannette), Jack Allen (Truscott), Ian Fleming (Talbot), Douglas Muir (Doctor), Sheila Raynor (Nurse), Courtney Hope (Mrs C. Smith), Nigel Neilson (Duty Officer), Darcy Conyers (Signal Operator), Kathleen Stuart (Receptionist), Anthony Miles (Sam), Laurie Taylor (O'Gorman), June Ashley, June Mitchell and Natasha Sokolova (Three Blondes) 
Stolen Face 1952, 72 min.

Production Company: Exclusive (Hammer)

Studio: Riverside

Producer: Anthony Hinds

Screenplay: Martin Berkley and Richard Landau, based on an original story by Alexander Paal and Steven Vas

Photography: Walter Harvey

Editor: Maurice Rootes

Music: Malcolm Arnold

Cast: Lizabeth Scott (Alice Brent and Lily B), Paul Henreid (Dr Philip Ritter), Andre Morell (David), John Wood (Jack), Mary Mackenzie (Lily A), Arnold Ridley (Russell), Susan Stephen (Betty), Cyril Smith (Alf), Diana Beaumont (May), Terence O'Regan (Pete), Russell Napier (Cutler), Ambrosine Phillpots (Miss Patton), Everly Gregg (Lady Harringay), Alexis France (Mrs Emmett), John Bull (Charles Emmett), Richard Wattis (Wentworth), Dorothy Bramhall (Miss Simpson), Janet Burnell (Maggie), Grace Gavin (Nurse), William Murray (Floor Walker), John Warren (Railway Guard), Anna Turner (Maid), Hal Osmond (Photographer), Philip Vickers (Soldier), James Valentine (Sailor), Howard Douglas (1st farmer), Brookes Turner (2nd farmer)

Distant Trumpet 1952, $63 \mathrm{~min}$.

Production company: Meridian

Producer: Derek Elphinstone

Screenplay: Derek Elphinstone

Photography: Gordon Lang

Editor: John Seabourne

Art director: George Jones

Music: David Jenkins

Cast: Derek Bond (David Anthony), Jean Patterson (Valerie Maitland), Derek Elphinstone (Richard Anthony), Grace Gavin (Miss Philips - 'Flips'), Anne Brooke (Beryl Jeffreys), Jean Webster-Brough (Mrs Waterhouse) 
Four Sided Triangle 1953, $81 \mathrm{~min}$.

Production company: Hammer

Studio: Bray

Producer: Michael Carreras and Alexander Paal

Screenplay: Paul Tabori and Terence Fisher from William F. Temple's novel

Photography: Reginald Wyer

Editor: Maurice Rootes

Art director: J. Elder Wills

Music: Malcolm Arnold

Cast: Barbara Payton (Lena and Helen), Stephen Murray (Bill), James Hayter (Dr Harvey), John Van Eyssen (Robin), Percy Marmont (Sir Walter), Kynaston Reeves (Lord Grant), Jennifer Dearman (Lena as a child), Glyn Dearman (Bill as a child), Sean Barrett (Robin as a child), John Stuart (Solicitor), Edith Saville (Lady Grant)

Mantrap 1953, 78 min.

Production company: Hammer

Studio: Bray

Producer: Michael Carreras and Alexander Paal

Screenplay: Paul Tabori and Terence Fisher, adapted by Tabori from Elleston Trevor's Queen in Danger

Photography: Reginald Wyer

Editor: Jim Needs

Art director: J. Elder Wills (credited simply as Elder Wills)

Music: Doreen Carwithen

Cast: Paul Henreid (Bishop), Lois Maxwell (Thelma), Kieron Moore (Speight), Hugh Sinclair (Jerrard), Kay Kendall (Vera), Lloyd Lamble (Frisnay), Anthony Forwood (Rex), Bill Travers (Victor), Mary Laura Wood (Susie), Liam Gaffney (Dorval), John Penrose (Du Vancet), Conrad Phillips (Barker), John Stuart (Doctor), Anna Turner (Marjorie), Christina Forrest (Joanna), Arnold Diamond (Alphonse), Jane Welsh (Laura), Geoffrey Murphy (Plain Clothes Man), Terry Carney (Detective), Sally Newland (Receptionist), Barbara Kowin (Fashion Commere) 
Spaceways 1953, $76 \mathrm{~min}$.

Production company: Hammer

Producer: Michael Carreras

Screenplay: Paul Tabori and Richard Landau from a radio play by

Charles Eric Maine

Photography: Reginald Wyer

Editor: Maurice Rootes

Art director: J. Elder Wills

Music: Ivor Slaney

Cast: Howard Duff (Stephen Mitchell), Eva Bartok (Lisa Frank), Andrew Osborn (Philip Crenshaw), Alan Wheatley (Smith), Philip Leaver (Dr Keppler), Michael Medwin (Toby Andrews), Cecile Chevreau (Vanessa), Anthony Ireland (General Hays), David Horne (Cabinet Minister)

Blood Orange 1953, $76 \mathrm{~min}$.

Production company: Hammer

Studio: Bray

Producer: Michael Carreras

Screenplay: Jan Read

Photography: Jimmy Harvey

Editor: Maurice Rootes

Art director: J. Elder Wills

Musical Director: Ivor Slaney

Cast: Tom Conway (Conway), Naomi Chance (Gina), Mila Parely (Helen Pascal), Eric Pohlman (Mercedes), Andrew Osborn (Captain Colin Simpson), Richard Wattis (Detective Inspector MacLeod), Margaret Halstan (Lady Marchant), Eileen Way (Mme Fernande), Michael Ripper (Eddie), Betty Cooper (Miss Betty), Thomas Heathcote (Detective Sergeant Jessup), Alan Rolfe (Inspector), Roger Delgado (Marlowe), Reed DeRoven (Heath), Delphi Lawrence (Chelsea), Christine Forrest (Blonde), Ann Hanslip (Jane), Davy Leon (George), Dorothy Robson (Seamstress), Leo Phillips (Harry), Robert Moore (Stevenson), Denis Cowles (Commissionaire), John H. Watson (Chauffeur), Cleo Rose (Vivian)

Note that some reference books list Tom Conway as playing himself in this film. However, it is clear from the film itself that Conway is playing a private detective who just happens 
to be called Tom Conway. I have no explanation for this little piece of bizarreness. Presumably it made sense at the time.

Three's Company 1953, $78 \mathrm{~min}$.

Fisher directed two segments - 'The Surgeon' and Take a Number' - while Charles Saunders directed 'The Scream'.

Production company: Douglas Fairbanks, Jnr

Screenplay: Richard Alan Simmons and Larry Marcus ('The Surgeon'), Larry Marcus (Take a Number')

Photography: Jimmy Wilson, Brendan Stafford

Editor: Inman Hunt, Sam Simmonds

Art direction: Norman Arnold

Music: Allan Gray

Three's Company was a compendium of material originally shot for the Douglas Fairbanks Presents TV series.

Face the Music 1954, $84 \mathrm{~min}$.

Production company: Hammer

Studio: Bray

Producer: Michael Carreras

Screenplay: Ernest Borneman from his novel

Photography: Jimmy Harvey

Editor: Maurice Rootes

Art director: J. Elder Wills

Musical directors: Ivor Slaney and Kenny Baker

Cast: Alex Nichol (James Bradley), Eleanor Summerfield (Barbara Quigley), John Salew (Max Margulis), Paul Carpenter (Johnny Sutherland), Geoffrey Keen (Maurice Green), Ann Hanslip (Maxine), Fred Johnson (Detective Sergeant MacKenzie), Arthur Lane (Jeff Colt), Martin Boddey (Inspector Mulrooney), Paula Byrne (Gloria Lewis Colt), Leo Phillips (Dresser), Freddie Tripp (Stage Manager), Ben Williams (Gatekeeper), Frank Birch (Trumpet Salesman), Jeremy Hawk (Recording Technician), James Carney (Mickey) and Kenny Baker's Dozen (Melvin Hayes, who would later play the young Baron in The Curse of Frankenstein appears briefly in an uncredited walkon part.) 
The Stranger Came Home 1954, $78 \mathrm{~min}$.

Production company: Exclusive (Hammer)

Producer: Michael Carreras

Screenplay: Michael Carreras from George Sanders' novel Stranger at Home

Photography: Jim Harvey

Editor: Bill Lenny

Art director: J. Elder Wills

Music: Leonard Salzedo, Ivor Slaney

Cast: Paulette Goddard (Angie), William Sylvester (Philip Vickers), Patrick Holt (Job Crandall), Paul Carpenter (Bill Saul), Alvys Maben (Joan Merrill), Russell Napier (Inspector Traherne)

Mask of Dust 1954, 79 min.

Production company: Hammer

Studio: Bray

Executive producer: Michael Carreras

Producer: Mickey Delamar

Screenplay: Richard Landau from a novel by Jon Manchip White Photography: Jimmy Harvey

Editor: Bill Lenny

Art director: J. Elder Wills

Music: Leonard Salzedo

Cast: Richard Conte (Peter Wells), Mari Aldon (Pat Wells), George Coulouris (Dallapiccola), Peter Illing (Bellario), Alec Mango (Guido Rizetti), Meredith Edwards (Lawrence), James Copeland (Johnny), Edwin Richfield, Richard Marner, Tim Turner, Jeremy Hawk and as themselves Stirling Moss, Reg Parnell, John Cooper, Alan Brown, Geoffrey Taylor, Leslie Marr

Final Appointment 1954, 69 min.

Production company: A. C. T. Films

Studio: Nettlefold

Producer: Francis Searle

Screenplay: Kenneth Hayles, adapted from the play Death Keeps a

Date by Sidney Nelson and Maurice Harrison

Photography: Jonah Jones 
Editor: John Ferris

Art director: C. P. Norman

Cast: John Bentley (Mike Billings), Eleanor Summerfield (Jenny), Hubert Gregg (Hartnell), Liam Redmond (Inspector Corcoran), Jean Lodge (Laura Robens), Meredith Edwards (Tom Martin), Sam Kydd (Vickery), Charles Farrell (Percy), Peter Bathurst (Harold Williams), Arthur Lowe (Barratt), Gerald Case (Australian Official), Jessica Cairns (War Office Typist), Tony Hilton (Jimmy), Henry De Bray (Restaurant Manager), John H. Watson (Police Sergeant)

Children Galore 1954, $60 \mathrm{~min}$.

Production company: Grendon Films

Studio: Brighton

Producer: Henry Passmore

Screenplay: John and Emery Bonett and Peter Plaskitt

Photography: Jonah Jones

Editor: Inman Hunter

Art director: John Elphick

Cast: Eddie Byrne (Zacky Jones), June Thorburn (Milly Ark), Betty Ann Davies (Mrs Ark), Richard Leach (Harry Bunnion), Marjorie Rhodes (Ada Jones), Jack McNaughton (Pat Ark), Violet Gould (Mrs Bunnion), Peter Evan Thomas (Lord Redscarfe), Marjorie Hume (Lady Redscarfe), Lucy Griffiths (Miss Prescott), Henry Caine (Bert Bunnion)

Murder by Proxy 1955, $87 \mathrm{~min}$.

Production company: Hammer

Producer: Michael Carreras

Screenplay: Richard Landau from a novel by Helen Nielsen

Photography: Jimmy Harvey

Editor: Maurice Rootes

Art director: Jim Elder Wills

Music: Ivor Slaney

Cast: Dane Clark (Casey Morrow), Belinda Lee (Phyllis Brunner), Eleanor Summerfield (Maggie Doone), Andrew Osborn (Lance Gordon), Betty Ann Davies (Alicia Brunner), Jill Melford (Miss Nardis), Harold Lang (Travis), Michael Golden (Inspector Johnson) 
The Flaw 1955, 61 min.

Production company: Cybex

Producer: Geoffrey Goodhart and Brandon Fleming

Screenplay: Brandon Fleming

Photography: Cedric Williams

Editor: Carmen Belaieff

Cast: John Bentley (Paul Oliveri), Donald Houston (John Millway), Rona Anderson (Monica), Doris Yorke (Mrs Bower), Tonia Bern (Vera), J. Trevor Davis (Sir George Bentham), Cecilia Cavendish (Lady Bentham)

Stolen Assignment 1955, $62 \mathrm{~min}$.

Production company: A. C. T. Films

Studio: Bray

Producer: Francis Searle

Screenplay: Kenneth Hayles from a story by Sidney Nelson and Maurice Harrison

Photography: James Harvey

Editor: John Pomeroy

Art director: William Kellner

Cast: John Bentley (Mike Billings), Hy Hazell (Jenny Drew), Eddie Byrne (Inspector Corcoran), Patrick Holt (Henry Crossley), Joyce Carey (Ida Garnett), Kay Callard (Stella Watson), Violet Gould (Mrs Hudson), Jessica Cairns (Marilyn Dawn), Charles Farrell (Percy Simpson), Michael Ellison (Danny Hudson), Desmond Rayner (John Smith), Graham Stuart (Coroner), Frank Forsyth (Dr Roberts), Clement Hamelin (Seth Makepeace), John H. Watson (Plain Clothes Detective Sergeant), Raymond Rollett (Desk Sergeant)

The Last Man to Hang? 1956, 75 min.

Production company: A. C. T. Films

Producer: John Gossage

Screenplay: Ivor Montagu and Max Trell, adapted by Gerald Bullett and Maurice Elvey from the novel The Jury by Gerald Bullett

Photography: Desmond Dickinson

Editor: Peter Taylor 
Art director: Alan Harris

Music: John Wooldridge

Cast: Tom Conway (Sir Roderick Strood), Elizabeth Sellers (Daphne Strood), Eunice Gayson (Elizabeth Anders), Freda Jackson (Mrs Tucker), Hugh Latimer (Mark Perryman), Raymond Huntley (Attorney General), Margaretta Scott (Mrs Cranshaw)

Kill Me Tomorrow 1957, 80 min.

Production company: Delta Films

Studio: Southall

Producer: Francis Searle

Screenplay: Robert Falconer and Manning O'Brine, from an original story by Robert Falconer

Photography: Geoffrey Faithful

Editor: Ann Chegwidden

Art director: Bernard Robinson

Music: Temple Abady

Cast: Pat O'Brien (Bart Crosbie), Lois Maxwell (Jill Brook), George Coulouris (Heinz Webber), Robert Brown (Steve), Ronald Adam (Brook), Richard Pasco (Dr Fisher), Wensley Pithey (Inspector Lane), Freddie Mills (Waxie) plus Tommy Steele

The Curse of Frankenstein 1957, $83 \mathrm{~min}$.

Production company: Hammer

Studio: Bray

Producer: Anthony Hinds

Screenplay: Jimmy Sangster

Photography: Jack Asher

Editor: James Needs

Production designer: Bernard Robinson

Music: James Bernard

Cast: Peter Cushing (Victor Frankenstein), Hazel Court (Elizabeth), Robert Urquhart (Paul Krempe), Christopher Lee (Creature), Melvyn Hayes (Young Victor), Valerie Gaunt (Justine), Paul Hardtmuth (Professor Bernstein), Noel Hood (Aunt), Fred Johnson (Grandpa), Claude Kingston (Little Boy), Alex Gallier (Priest), Michael Mulcaster (Warder), Andrew Leigh (Burgomaster), Ann Blake (Wife), Sally Walsh (Young Elizabeth), Middleton Woods (Lecturer), Raymond Ray (Uncle) 
Dracula 1958, $82 \mathrm{~min}$.

Production company: Hammer

Studio: Bray

Producer: Anthony Hinds

Screenplay: Jimmy Sangster

Photography: Jack Asher

Supervising editor: James Needs

Production designer: Bernard Robinson

Music: James Bernard

Cast: Peter Cushing (Dr Van Helsing), Christopher Lee (Dracula), Michael Gough (Arthur), Melissa Stribling (Mina), Carol Marsh (Lucy), Olga Dickie (Gerda), John Van Eyssen (Jonathan), Valerie Gaunt (Vampire Woman), Janine Faye (Tania), Barbara Archer (Inga), Charles Lloyd Pack (Dr Seward), George Merritt (Policeman), George Woodbridge (Landlord), George Benson (Official), Miles Malleson (Undertaker), Geoffrey Bayldon (Porter), Paul Cole (Lad)

The Revenge of Frankenstein 1958, $89 \mathrm{~min}$.

Production company: Hammer

Studio: Bray

Producer: Anthony Hinds

Screenplay: Jimmy Sangster, additional dialogue by Hurford Janes Photography: Jack Asher

Supervising editor: James Needs

Production designer: Bernard Robinson

Music: Leonard Salzedo

Cast: Peter Cushing (Victor Stein/Frankenstein), Francis Matthews (Dr Hans Kleve), Eunice Gayson (Margaret), Michael Gwynn (Karl), John Welsh (Bergman), Lionel Jeffries (Fritz), Oscar Quitak (Dwarf), Richard Wordsworth (Up Patient), Charles Lloyd Pack (President), John Stuart (Inspector), Arnold Diamond (Molke), Margery Gresley (Countess Barscynska), Anna Walmsley (Vera Barscynska), George Woodbridge (Janitor), Michael Ripper (Kurt), Ian Whittaker (Boy), Avril Leslie (Girl) 
The Hound of the Baskervilles 1959, $87 \mathrm{~min}$.

Production company: Hammer

Studio: Bray

Producer: Anthony Hinds

Screenplay: Peter Bryan

Photography: Jack Asher

Supervising editor: James Needs

Production designer: Bernard Robinson

Music: James Bernard

Cast: Peter Cushing (Sherlock Holmes), Andre Morell (Dr Watson), Christopher Lee (Sir Henry), Marla Landi (Cecile), David Oxley (Sir Hugo), Francis de Wolff (Dr Mortimer), Miles Malleson (Bishop), Ewen Solon (Stapleton), John Le Mesurier (Barrymore), Helen Goss (Mrs Barrymore), Sam Kydd (Perkins), Michael Hawkins (Lord Caphill), Judi Moyens (Servant Girl), Michael Mulcaster (Convict), David Banks (Servant)

The Man Who Could Cheat Death 1959, 83 min.

Production company: Hammer

Studio: Bray

Producer: Anthony Hinds

Screenplay: Jimmy Sangster from Barre Lyndon's play The Man in Half Moon Street

Photography: Jack Asher

Supervising editor: James Needs

Production designer: Bernard Robinson

Music: Richard Bennett

Cast: Anton Diffring (Georges), Hazel Court (Janine), Christopher Lee (Pierre), Arnold Marie (Ludwig), Delphi Lawrence (Margo), Francis de Wolff (Legris), Gerda Larsen (Street Girl)

The Mummy 1959, 88 min.

Production company: Hammer

Studio: Bray (with additional material shot at Shepperton)

Producer: Michael Carreras

Screenplay: Jimmy Sangster

Photography: Jack Asher 
Supervising editor: James Needs

Production designer: Bernard Robinson

Music: Franz Reizenstein

Cast: Peter Cushing (John Banning), Christopher Lee (The Mummy/ Kharis), Yvonne Furneaux (Isobel/Ananka), Eddie Byrne (Inspector Mulrooney), Felix Aylmer (Stephen Banning), Raymond Huntley (Joseph Whemple), George Pastell (Mehemet Bey), Michael Ripper (Poacher), George Woodbridge (Police Constable), Harold Goodwin (Pat), Denis Shaw (Mike), Gerald Lawson (Irish Customer), Willoughby Gray (Dr Reilly), John Stuart (Coroner), David Browning (Police Sergeant), Frank Sieman (Bill), Stanley Meadows (Attendant), Frank Singuineau (Head Porter)

The Stranglers of Bombay 1959, $80 \mathrm{~min}$.

Production company: Hammer

Studio: Bray

Producer: Anthony Hinds

Screenplay: David Z. Goodman

Photography: Arthur Grant

Supervising editor: James Needs

Production designer: Bernard Robinson

Music: James Bernard

Cast: Guy Rolfe (Lewis), Allan Cuthbertson (Connaught-Smith), Andrew Cruickshank (Henderson), Marne Maitland (Patel Shari), Jan Holden (Mary), George Pastell (High Priest), Paul Stassino (Silver), David Spenser (Gopali), Tutte Lemkow (Ram Das), Roger Delgado (Bundar), John Harvey (Burns), Michael Nightingale (Flood), Marie Devereux (Karim)

The Two Faces of Dr Jekyll 1960, 88 min.

Production company: Hammer

Studio: Bray (with additional material shot at Elstree)

Producer: Michael Carreras

Screenplay: Wolf Mankowitz

Photography: Jack Asher

Supervising editor: James Needs

Production designer: Bernard Robinson

Music and songs: Monty Norman and David Heneker 
Cast: Paul Massie (Jekyll/Hyde), Dawn Addams (Kitty), Christopher Lee (Paul Allen), David Kossoff (Litauer), Francis de Wolff (Inspector), Norma Marla (Maria), Magda Miller (Sphinx Girl), William Kendall (Clubman), Helen Goss (Nannie) plus an uncredited appearance by Oliver Reed as a nightclub customer.

The Brides of Dracula 1960, $85 \mathrm{~min}$.

Production company: Hammer

Studio: Bray

Producer: Anthony Hinds

Screenplay: Jimmy Sangster, Peter Bryan and Edward Percy

Photography: Jack Asher

Supervising editor: James Needs

Production designer: Bernard Robinson

Music: Malcolm Williamson

Cast: Peter Cushing (Dr Van Helsing), Martita Hunt (Baroness Meinster), Yvonne Monlaur (Marianne), Freda Jackson (Greta), David Peel (Baron Meinster), Miles Malleson (Dr Tobler), Henry Oscar (Herr Lang), Mona Washbourne (Frau Lang), Andree Melly (Gina), Victor Brooks (Hans), Fred Johnson (Cure), Michael Ripper (Coachman), Norman Pierce (Landlord), Vera Cook (Landlord's wife), Marie Devereux (Village Girl), Michael Mulcaster (Latour)

The Sword of Sherwood Forest 1960, $80 \mathrm{~min}$.

Production company: Hammer/Yeoman

Studio: Ardmore Studios, Ireland

Producer: Sidney Cole and Richard Greene

Screenplay: Alan Hackney

Photography: Ken Hodges

Editor: James Needs

Art director: John Stoll

Music: Alun Hoddinott

Cast: Richard Greene (Robin Hood), Peter Cushing (Sheriff of Nottingham), Niall McGinnis (Friar Tuck), Richard Pasco (The Earl of Newark), Jack Gwillim (Hubert Walter, Archbishop of Canterbury), Sarah Branch (Maid Marian), Nigel Green (Little John), Vanda Godsell (Prioress), Edwin Richfield (Sheriffs Lieutenant), Charles Lamb (Old Bowyer), 
Dennis Lotis (Allan A' Dale); uncredited - Derren Nesbitt (Martin), Richard Crean (Ollerton), Oliver Reed (Melton), Adam Kean (Retford), James Neylin (Roger)

The Curse of the Werewolf 1961, $88 \mathrm{~min}$.

Production company: Hammer

Studio: Bray

Producer: Anthony Hinds

Screenplay: John Elder

Photography: Arthur Grant

Supervising editor: James Needs

Production designer: Bernard Robinson

Music: Benjamin Frankel

Cast: Clifford Evans (Alfredo), Oliver Reed (Leon), Yvonne Romain (Servant Girl), Catherine Feller (Cristina), Anthony Dawson (The Marques Siniestro), Josephine Llewellyn (The Marquesa), Richard Wordsworth (The Beggar), Hira Talfrey (Teresa), Justin Walters (Young Leon), John Gabriel (The Priest), Warren Mitchell (Pepe Valiente), Anne Blake (Rosa Valiente), George Woodbridge (Dominique), Michael Ripper (Old Soak), Ewen Solon (Don Fernando), Peter Sallis (Don Enrique), Martin Matthews (Jose), David Conville (Rico Gomez), Denis Shaw (Gaoler), Charles Lamb (Chef), Serafma di Leo (Senora Zumara), Sheila Brennan (Vera), Joy Webster (Isabel), Renny Lister (Yvonne)

The Phantom of the Opera 1962, $84 \mathrm{~min}$.

Production company: Hammer

Studio: Bray

Producer: Anthony Hinds

Screenplay: John Elder, from the novel (or the composition as the film credits put it) by Gaston Leroux

Photography: Arthur Grant

Supervising editor: James Needs

Production designer: Bernard Robinson

Music: Edwin Astley

Cast: Herbert Lom (The Phantom/Professor Petrie), Heather Sears (Christine), Edward De Souza (Harry), Thorley Walters (Latimer), Michael Gough (Ambrose), Harold Goodwin (Bill), Martin Miller (Rossi), Liane Aukin (Maria), Sonya Cordeau 
(Yvonne), Marne Maitland (Xavier), Miriam Karlin (Charwoman), Patrick Troughton (Ratcatcher), Renee Houston (Mrs Tucker), Keith Pyott (Weaver), John Harvey (Sergeant Vickers), Michael Ripper (1st Cabby), Miles Malleson (2nd Cabby), Ian Wilson (Dwarf)

Sherlock Holmes und das Halsband des Todes 1962, 86 min.

(co-directed - according to the credits at least - by Frank Winterstein)

Production company: CCC (Berlin)/ Criterion Film (Paris)/ INCEI Film (Rome)

Studio: CCC Studios, Berlin

Producer: Artur Brauner

Screenplay: Curt Siodmak

Photography: Richard Angst

Editor: Ira Oberberg

Art Director: Paul Markwitz

Music: Martin Slavin

Cast: Christopher Lee (Sherlock Holmes), Thorley Walters (Dr Watson), Hans Söhnker (Professor Moriarty), Hans Nielsen (Inspector Cooper), Senta Berger (Ellen Blackburn), Ivan Desny (Paul King), Wolfgang Lukschy (Peter Blackburn), Leon Askin (Charles), Edith Schutlze-Westrum (Mrs Hudson), Bernard Lajarrige (French Police Inspector), Bruno Panthel, Heinrich Gies, Linda Sini, Roland Armontel, Danielle Argence, Franco Giacobini, Waldermar Frahm, Renate Hütter, Max Strassberg

The Horror of it All 1964, $75 \mathrm{~min}$.

Production company: Lippert

Producer: Robert L. Lippert

Screenplay: Ray Russell

Photography: Arthur Lavis

Editor: Robert Winter

Art director: Harry White

Music: Douglas Gamley

Cast: Pat Boone (Jack Robinson), Erica Rogers (Cynthia), Dennis Price (Cornwallis), Andree Melly (Natalia), Valentine Dyall (Reginald), Jack Bligh (Percival), Erik Chitty (Grandpapa), Archie Duncan (Muldoon), Oswald Lawrence (Young Doctor) 
The Gorgon 1964, $83 \mathrm{~min}$.

Production company: Hammer

Studio: Bray

Producer: Anthony Nelson Keys

Screenplay: John Gilling (with an uncredited rewrite by Anthony

Hinds), from an original story by J. Llewellyn Devine

Photography: Michael Reed

Supervising editor: James Needs

Production designer: Bernard Robinson

Music: James Bernard

Leading players: Peter Cushing (Dr Namaroff), Christopher Lee (Professor Meister), Richard Pasco (Paul Heitz), Barbara Shelley (Carla Hoffmann), Michael Goodliffe (Professor Heitz), Patrick Troughton (Kanof), Jack Watson (Ratoff), Jeremy Longhurst (Bruno Heitz), Toni Gilpin (Sascha), Redmond Phillips (Hans), Joseph O'Conor (Coroner), Alister Williamson (Cass), Joyce Hemson (Martha), Michael Peake (Policeman), Sally Nesbitt (Nurse), Prudence Hyman (Chatelaine - a lady of the castle. This is, of course, a sly joke. In fact she plays Megaera.)

The Earth Dies Screaming 1964, $62 \mathrm{~min}$.

Production company: Lippert

Studio: Shepperton

Producer: Robert L. Lippert

Screenplay: Henry Cross

Photography: Arthur Lavis

Supervising editor: Robert Winter

Art director: George Provis

Music: Elisabeth Lutyens

Cast: Willard Parker (Jeff Nolan), Virginia Field (Peggy), Dennis Price (Taggart), Thorley Walters (Otis), Vanda Godsell (Violet), David Spenser (Mel), Anna Palk (Lorna)

Dracula - Prince of Darkness 1966, 90 min.

Production company: Hammer

Studio: Bray

Producer: Anthony Nelson Keys

Screenplay: John Sansom (Jimmy Sangster) 
Photography: Michael Reed

Supervising editor: James Needs

Production designer: Bernard Robinson

Music: James Bernard

Cast: Christopher Lee (Dracula), Barbara Shelley (Helen), Andrew Keir (Father Sandor), Francis Matthews (Charles), Suzan Farmer (Diana), Charles Tingwell (Alan), Thorley Walters (Ludwig), Philip Latham (Klove), Walter Brown (Brother Mark), George Woodbridge (Landlord), Jack Lambert (Brother Peter), Philip Ray (Priest), Joyce Hemson (Mother), John Maxim (Coach Driver)

Island of Terror 1966, $89 \mathrm{~min}$.

Production company: Planet

Studio: Pinewood

Producer: Tom Blakeley

Screenplay: Edward Andrew Mann and Alan Ramsen

Photography: Reg Wyer

Editor: Thelma Connell

Art director: John St John Earl

Music: Malcolm Lockyer

Cast: Peter Cushing (Dr Stanley), Edward Judd (Dr David West), Carole Gray (Toni Merrill), Eddie Byrne (Dr Landers), Sam Kydd (Constable Harris), Niall MacGinnis (Mr Campbell), James Caffrey (Argyle), Liam Gaffney (Bellows), Roger Heathcote (Dunley), Keith Bell (Halsey), Shay Gorman (Morton), Peter Forbes Robertson (Dr Phillips), Richard Bidlake (Carson), Joyce Hemson (Mrs Bellows), Edward Ogden (Helicopter Pilot)

Frankenstein Created Woman 1967, $86 \mathrm{~min}$.

Production company: Hammer

Studio: Bray

Producer: Anthony Nelson Keys

Screenplay: John Elder

Photography: Arthur Grant

Supervising editor: James Needs

Production designer: Bernard Robinson

Music: James Bernard 
Cast: Peter Cushing (Baron Frankenstein), Susan Denberg (Christina), Thorley Walters (Doctor Hertz), Robert Morris (Hans), Duncan Lamont (The Prisoner), Peter Blythe (Anton), Barry Warren (Karl), Derek Fowlds (Johann), Alan MacNaughtan (Kleve), Peter Madden (Chief of Police), Philip Ray (Mayor), Ivan Beavis (Landlord), Colin Jeavons (Priest), Bartlett Mullins (Bystander), Alec Mango (Spokesman)

Night of the Big Heat 1967, $94 \mathrm{~min}$.

Production company: Planet

Studio: Pinewood

Producer: Tom Blakeley

Screenplay: Ronald Liles from John Lymington's novel - additional scenes and dialogue by Pip and Jane Baker

Photography: Reg Wyer

Editor: Rod Keys

Art director: Alex Vetchinsky

Music: Malcolm Lockyer

Cast: Christopher Lee (Godfrey Hanson), Patrick Allen (Jeff Callum), Peter Cushing (Dr Vernon Stone), Jane Merrow (Angela Roberts), Sarah Lawson (Frankie Callum), William Lucas (Ken Stanley), Kenneth Cope (Tinker Mason), Percy Herbert (Gerald Foster), Tom Heathcote (Bob Hayward), Anna Turner (Stella Hayward), Jack Bligh (Ben Siddle), Sidney Bromley (Old Tramp), Barry Halliday (Radar Operator)

The Devil Rides Out 1968, $95 \mathrm{~min}$.

Production company: Hammer

Studio: Elstree

Producer: Anthony Nelson Keys

Screenplay: Richard Matheson, from the novel by Dennis Wheatley

Photography: Arthur Grant

Supervising editor: James Needs

Production designer: Bernard Robinson

Music: James Bernard

Cast: Christopher Lee (Due de Richleau), Charles Gray (Mocata), Nike Arrighi (Tanith), Leon Greene (Rex), Patrick Mower (Simon), Gwen Ffrançon-Davis (Countess), Sarah Lawson (Marie), Paul Eddington (Richard), Rosalyn Landor (Peggy), Russell Waters (Malin) 
Frankenstein Must Be Destroyed 1969, 97 min.

Production company: Hammer

Studio: Elstree

Producer: Anthony Nelson Keys

Screenplay: Bert Batt, from a story by Anthony Nelson Keys and Bert Batt

Photography: Arthur Grant

Supervising editor: James Needs

Production designer: Bernard Robinson

Music: James Bernard

Cast: Peter Cushing (Baron Frankenstein), Veronica Carlson (Anna), Freddie Jones (Professor Richter), Simon Ward (Karl), Thorley Walters (Inspector Frisch), Maxine Audley (Ella Brandt), George Pravda (Brandt), Geoffrey Bayldon (Police Doctor), Colette O'Neil (Mad Woman), Frank Middlemass, George Belbin, Norman Shelley, Michael Gover (Guests), Peter Copley (Principal), Jim Collier (Dr. Heidecke), Allan Surtees, Windsor Davies (Police Sergeants), Harold Goodwin (Burglar)

\section{Frankenstein and the Monster from Hell 1974, $99 \mathrm{~min}$.}

Production company: Hammer

Studio: Elstree

Producer: Roy Skeggs

Screenplay: John Elder

Photography: Brian Probyn

Editor: James Needs

Art director: Scott MacGregor

Music: James Bernard

Cast: Peter Cushing (Baron Frankenstein), Shane Briant (Simon), Madeline Smith (Sarah), David Prowse (Monster), John Stratton (Asylum Director), Michael Ward (Transvest), Elsie Wagstaff (Wild One), Norman Mitchell (Police Sergeant), Clifford Mollison (Judge), Patrick Troughton (Bodysnatcher), Philip Voss (Ernst), Chris Cunningham (Hans), Charles LloydPack (Professor Durendel), Lucy Griffiths (Old Kay), Bernard Lee (Tarmut), Sydney Bromley (Muller), Andrea Lawrence (Brassy Girl), Jerold Wells (Landlord), Sheila D’Union (Gerda), Mischa de la Motte (Twitch), Norman Atkyns (Smiler), Victor Woolf (Letch), Winifred Sabine (Mouse), Janet Hargreaves (Chatter), Peter Madden (Coach Driver) 